\title{
1 Analysis of small-scale structures in lidar observations of noctilucent clouds using a pattern recognition method
}

\author{
C. Ridder ${ }^{a, *}$, G. Baumgarten ${ }^{a, *}$, J. Fiedler ${ }^{\mathrm{a}}$, F-J. Lübken ${ }^{\mathrm{a}}$, G. Stober ${ }^{\mathrm{a}}$ \\ ${ }^{a}$ Leibniz-Institute of Atmospheric Physics at the Rostock University, Schlossstraße 6, 18225 Kühlungsborn, Germany
}

Noctilucent clouds (NLC) have been observed with the ALOMAR Rayleigh/Mie/Raman lidar at $69^{\circ} \mathrm{N}$ using a temporal resolution of 30 s since 2008 . We present an approach to identify and analyze the localized small scale wave structures of the varying altitude of the NLC layers in the range of 5-30 min that may be caused by gravity waves. Small scale gravity waves breaking in the mesopause region contribute notably to the momentum flux but are difficult to observe and to characterize. The approach is based on a template matching method using generalized structures to be identified in the NLC observations. The new method permits the identification of structures that are present in NLC only for a time too short to appear in a Fourier or wavelet spectrum. Without the need for a continuous time series the method can handle multiple NLC layers and data gaps. In the $2000 \mathrm{~h}$ of NLC data from the years 2008-2015, we find almost 5000 single wave structures with a total length of $738 \mathrm{~h}$. The structures are found on average $400 \mathrm{~m}$ below the NLC centroid altitude and a large number of the structures has a length at the lower limit of $5 \mathrm{~min}$. With the background wind from the meteor radar near ALOMAR a horizontal scale is estimated based on the length of the individual structures. The distribution of horizontal scales shows a peak of wave structures at $15-20 \mathrm{~km}$ in accordance with the horizontal wavelengths found by ground-based camera observations of NLC.

Key words: Noctilucent clouds, Polar mesospheric clouds, Lidar, Gravity waves, Dynamics

\section{Introduction}

Noctilucent clouds (NLC) are mesospheric clouds first observed in 1885 (e.g. Jesse, 1885; Backhouse 1885; Leslie, 1885), that exist at an altitude of about $83 \mathrm{~km}$ in the Northern Hemisphere and are composed of ice particles (e.g. Jesse, 1896; von Zahn et al., 1998; Lübken et al., 2008; Hervig et al., 2001). They are an important tracer for the processes in the mesosphere, an altitude region that is difficult to study otherwise. Gravity waves of different scales influence the brightness of the clouds and generate their characteristic, highly structured appearance that is visible from the ground (Hines, 1968; Fritts et al., 1993). Wave breaking near the mesopause is a crucial driver of atmospheric dynamics (e.g. Holton, 1983; Geller, 1983), but their scales and propagation properties are still not sufficiently known. Regarding small scales (here: horizontal scales of up to $100 \mathrm{~km}$ and temporal scales up to $30 \mathrm{~min}$ ) the observation methods of NLC are limited and even high

\footnotetext{
* Corresponding author

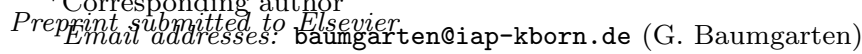


resolution ice models provide no sufficient resolution (e.g. Chandran et al., 2012; Kiliani et al., 2013). Rapp et al. (2002) simulated the influence of gravity waves on NLC, but used a minimum period of $1 \mathrm{~h}$ which is the upper limit for the waves considered here. However, it is believed that such small-scale gravity waves contribute a large fraction of the total momentum flux generated by gravity waves in the mesopause region (Fritts et al., 2014).

The ice particles in this height region are therefore a most welcome tracer, that is easier to observe than the variations in the atmospheric background. They are visible by naked eye and with ground based cameras while the sun is just below the horizon and may also be observed by lidar independent of daytime. Observed from space, the clouds are called polar mesospheric clouds (PMC). Many wave patterns are visible in observations of all these instruments on different scales and constitute a distinctive characteristic of NLC (Witt, 1962; Pautet et al., 2011; Demissie et al., 2014; Chandran et al., 2009; Kaifler et al., 2013). Recently, high resolution observations by camera from ground and stratospheric balloons became available, which resolve gravity waves and their transition to turbulence on scales down to a few meters (Baumgarten and Fritts, 2014; Miller et al., 2015). Hoffmann et al. (2008) simulated the effect of small scale gravity waves with periods of $1 \mathrm{~h}$ on polar mesospheric summer echoes (PMSE) and concluded that gravity waves cause the vertical wave motions that are observed in PMSE and NLC.

While ground-based camera observations are possible only during a limited time period after sunset, lidar measurements allow for continuous measurements independent of daylight, limited by weather conditions only. Lidar observations yield the absolute NLC backscatter coefficient and also the particle number density, shape and size (Hansen et al., 1989; von Cossart et al., 1999; Baumgarten et al., 2002). Instead of a horizontally resolved image as with a camera, the result is a vertical brightness profile in a fixed measurement volume. Previous studies of vertical oscillations rely on a wavelet analysis of the centroid altitude of the NLC brightness and were also applied separately for the sub-layers in the NLC (Kaifler et al., 2013).

We use an alternative approach targeting specifically the small scale wave structures shorter than 30 min and appearing only for a few oscillations. We present an algorithm based on pattern recognition and show how it compares to wavelet analysis. Pattern recognition is often used for machine vision applications such as face recognition (e.g Viola and Jones, 2004). However such techniques are most successful if the problem can be generalized so that the computational effort is manageable (Bishop, 2006). The approach used here is a template matching algorithm. Template matching is applied in object recognition or face recognition, often combined with further, more sophisticated methods or a learning algorithm that are not necessary in this application (e.g Brunelli and Poggio, 1993).

The NLC observations from 2008 to 2015 by the ALOMAR RMR lidar are analyzed and the results for temporal and horizontal scales of wave structures retrieved by template matching using generalized structures will be shown. 


\section{Instruments and data base}

\subsection{ALOMAR RMR-Lidar}

The ALOMAR Rayleigh/Mie/Raman-Lidar is a twin lidar system located at $69^{\circ} \mathrm{N}$, where NLC are detected during $43 \%$ of the time in the summer season (Fiedler et al., 2003). The sophisticated spatial and spectral filtering of the received signal allows for measurements throughout summer under daylight conditions. A detailed description of the instrument is given by von Zahn et al. (2000). The extraction of NLC data from the raw lidar signal which yields the NLC backscatter coefficient $\beta_{\mathrm{NLC}}(t, z)$ as a function of altitude and time is described by Fiedler et al. (2009). We follow previous conventions and refer to the NLC backscatter coefficient as NLC brightness. Since 2008, high resolution data of the NLC brightness with a temporal resolution of $30 \mathrm{~s}$ and a vertical resolution of $40 \mathrm{~m}$ is available. Before further processing the data is smoothed with a $2 \mathrm{~d}$ Gaussian Kernel with $\sigma=40 \mathrm{~m}$ and $30 \mathrm{~s}$ respectively. During the NLC season (from 1 June to 15 August at this latitude), the lidar is operated whenever permitted by weather conditions. In the years 2008-2015, the two systems together recorded more than $4500 \mathrm{~h}$ of high-resolution measurements during the summer season. A total of $1900 \mathrm{~h}$ of NLC were measured during this time. The two laser beams of the system can be tilted up to $30^{\circ}$ from zenith into the northwest and the southeast quadrant respectively. In $15 \%$ of the measurements, both laser beams are pointing vertically or only one system was in operation. During the remaining $85 \%$ of the measurements, the laser beams were tilted by $20^{\circ}$ to the north and east, leading to a separation of the measurement volumes of about $40 \mathrm{~km}$ at the altitude of the NLC. At small scales, the NLC show significant differences at simultaneous measurements in the two systems, so the measurements by the different systems are considered as independent for this analysis.

\subsection{Andenes Meteor Radar}

The Andenes meteor radar is located approximately $2 \mathrm{~km}$ northeast of the ALOMAR observatory. The system transmits with a peak power of $30 \mathrm{~kW}$ at a frequency of $32.55 \mathrm{MHz}$ and detects between 12000 and 18000 specular meteor trails per day during summer. More details about the radar system can be found in Hocking et al. (2001).

The meteor radar winds used in this study are processed with a temporal resolution of 30 minutes by moving a one hour window. The vertical resolution of $1 \mathrm{~km}$ was obtained using a $3 \mathrm{~km}$ oversampling window centered at each height. The 3D winds (zonal, meridional and vertical) are obtained by fitting each radial velocity measurement weighted by its statistical uncertainty. The algorithm includes a regularization scheme to get a smooth solution in space (vertical) and time $d u / d t=$ const, $d v / d t=$ const, $d u / d z=$ const and $d v / d z=$ const. Additionally we regularize the vertical wind to be very small $w \approx 0$, which seems to be justified considering the large measurement volume of $600 \mathrm{~km}$ in diameter of the meteor radar. This procedure leads to statistical uncertainties in the horizontal wind components in the order of $1-5 \mathrm{~m} / \mathrm{s}$. The 
larger uncertainties usually occur at the upper and lower boundaries of the meteor layer due to the smaller number of meteor detections.

\section{Data analysis}

The results presented here are based on a new approach to identify wave structures that are found in NLC lidar data. This approach allows to quantify and characterize these structures. An analysis of the waves in NLC lidar data needs to take into account two aspects of the data: (1) The data is two-dimensional (NLC brightness as function of time and altitude). While a reduction to a single time series is for some parts sufficient to describe the dynamics of the NLC layer, the two-dimensional information is lost. (2) Small scale wave structures are visible only for a short period of time, the local analysis of the data is therefore important. The new method imitates a manual identification of $2 \mathrm{~d}$ wave structures (altitude profile of brightness in time), which would be possible in theory, but due to the large NLC data set is not reasonable nor reproducible. The goal is therefore to automatically identify structures in NLC that are evident in a visual two-dimensional representation of the NLC brightness in time and altitude.

To find typical wave-like structures, images of five variations of a "V"-shaped oscillations were chosen as template images. Together with their " $\Lambda$ "-shaped inverse, they represent the common wave features in NLC observations, the vertical oscillation of a bright layer on a darker background. Such a reduction of the task to the identification of simplified structures (generalization of the problem) is a typical approach in pattern recognition (Bishop, 2006). The analysis method uses a pattern matching algorithm to identify these templates in the NLC data using the cross-correlation between the template images at different sizes and the NLC data. The template images represent only a single oscillation, such that structures that appear only for a few or a single oscillation in the data will be detected. With this approach several difficulties arising with the traditional methods are avoided:

1. With the possibly large vertical extent of the NLC, a one-dimensional time series describes the NLC dynamics only insufficiently, especially for NLC featuring multiple layers (Fiedler et al., 2009; Kaifler et al., 2013).

2. Due to the changing weather conditions NLC data are typically affected by interruptions, ranging from minutes to hours. This limits a wavelet analysis to the data unaffected by gaps or requires interpolations which introduce artificial effects.

3. Sometimes very short wavelike features occur only for one or two cycles. They are suppressed in a global spectrum and are not significant in the local wavelet power spectrum (Kaifler et al., 2013).

The performance of the pattern recognition compared to a wavelet analysis is studied in detail in Section 3.3 

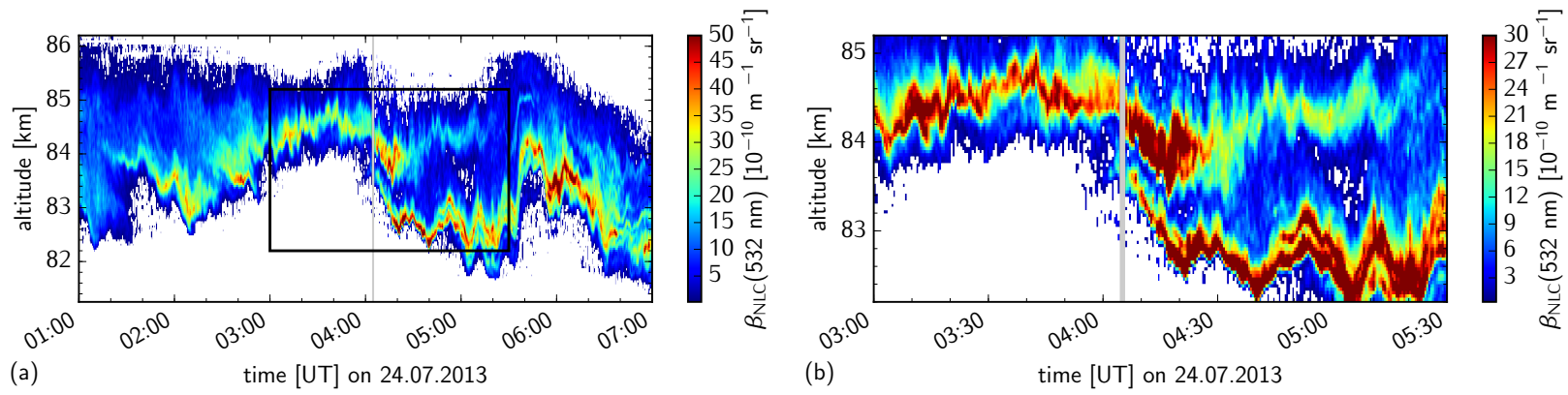

Figure 1: Lidar observation of NLC brightness on July 24, 2013 above ALOMAR. (a): $6 \mathrm{~h}$ time span, (b): $2.5 \mathrm{~h}$ time span. The time and altitude range plotted in panel (a) is indicated by a black rectangle in panel (b). Gray bars indicate times without measurements.
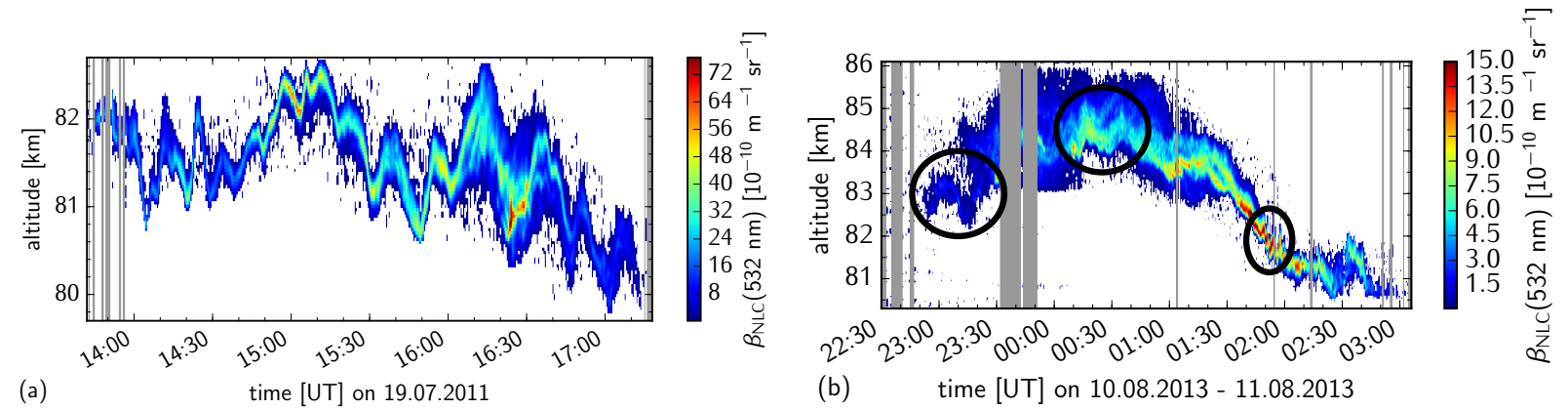

Figure 2: NLC observed on July 19, 2011 (a) and August 10, 2013 (b). Gray bars indicate times without measurements. Black circles in panel (b) indicate wavelike structures

\subsection{NLC data}

The ALOMAR RMR lidar observations show the temporal evolution of the NLC brightness in a fixed volume in the altitude range from 78 to $90 \mathrm{~km}$. Fig. 1 shows an example of the typical structures seen by lidar in NLC for a case with many wave features. The NLC is of moderate brightness (about $5-10 \cdot 10^{-10} \mathrm{~m}^{-1} \mathrm{sr}^{-1}$ ) over most of the altitude range except one thin, very bright layer of more than $25 \cdot 10^{-10} \mathrm{~m}^{-1} \mathrm{sr}^{-1}$, that splits into two layers after 04:00 UT.

While variations in the brightness of the NLC are visible, the most evident wave structures are those formed by the bright NLC layer. This layer features many small scale structures of 5-30 min.

In some cases the NLC may consist of only a thin structured layer, as it is the case for the NLC shown in Fig. 2a. This is one of the few cases where the small-scale $(\sim 15 \mathrm{~min})$ structures extend over several hours (see Fig. 2a). More often they appear only for a single or two oscillations as shown in Fig. 2p, where the circles indicate the presence of small scale wave structures. 


\subsection{Analysis: Pattern recognition}

To find wave structures in the two-dimensional NLC data, we use a template matching approach using template images that correspond to the generalized structures. The matchTemplate function from the Open Source Computer Vision Library (OpenCV) (Itseez, 2015) is used to calculate the normalized crosscorrelation between the template $(T)$ and the representation of the data $(I)$ :

$$
R(x, y)=\frac{\sum_{x^{\prime}, y^{\prime}}\left(T^{\prime}\left(x^{\prime}, y^{\prime}\right) I^{\prime}\left(x+x^{\prime}, y+y^{\prime}\right)\right)}{\sqrt{\sum_{x^{\prime}, y^{\prime}} T^{\prime}\left(x^{\prime}, y^{\prime}\right)^{2} \sum_{x^{\prime}, y^{\prime}} I^{\prime}\left(x+x^{\prime}, y+y^{\prime}\right)^{2}}},
$$

where $x$ and $y$ denote the time and altitude coordinates of the cross-correlation matrix $R, x^{\prime}$ and $y^{\prime}$ the coordinates of the template image. The brightness of the template is normalized by subtracting the mean brightness of the template $\bar{T}$

$$
T^{\prime}\left(x^{\prime}, y^{\prime}\right)=T\left(x^{\prime}, y^{\prime}\right)-\bar{T}
$$

Similarly, the brightness of the data portion at the position and of the size of the template is normalized:

$$
I^{\prime}\left(x+x^{\prime}, y+y^{\prime}\right)=I\left(x+x^{\prime}, y+y^{\prime}\right)-\bar{I}
$$

The result is a matrix with the cross-correlation coefficient of the template with the image at each possible template position. Local maxima of the cross-correlation coefficient are considered as successful matches if they exceed a fixed threshold. To account for the different periods and amplitudes of the wave structures in the NLC observations the correlation matrix is calculated for templates that are stretched in height and length independently. The entire algorithm is repeated for the different "V"-shaped templates. Since this might result in multiple matches for the same wave structure, a further multiple-match elimination is needed, which is discussed in detail below. The same procedure is then applied a second time, with all templates rotated by $180^{\circ}$ to find the corresponding inverse, " $\Lambda$ "-shaped oscillations.

The template matching analysis relies on several parameters that determine the final results. The values were chosen empirically based on a subset of the total data base (data from the year 2014 and random samples of the previous years to verify the results for different lidar sensitivities). The critical parameters are listed below, followed by a discussion of their influence and the value that was used:

1. selection of templates

2. size constraints for the templates

3. cross-correlation coefficient threshold that determines a successful match

4. minimum signal-to-noise ratio (SNR) for each match

5. multiple match elimination 


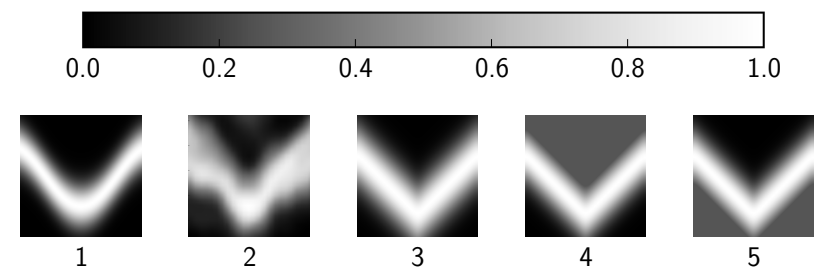

Figure 3: Template images used for the wave structure detection. The different templates cover different characteristic structures in the NLC data. The grey scale represents the relative brightness, the horizontal represents the time axis while the vertical represents the altitude axis of the actual data to be matched. The vertical brightness profile is given by a Gaussian (except for template 2).

\section{Selection of templates}

To find typical wave-like structures, five variations of a "V"-shaped oscillations were chosen. Together with their " $\Lambda$ "-shaped inverse, they represent the common wave feature in NLC observations, the vertical oscillation of a bright layer on a darker background. This structure represents only a single oscillation, such that structures that appear only for a few or a single oscillation in the data will still be detected. The different templates shown in Fig. 3 cover several characteristic structures. The bright layer is given by a sine function for the first template and targets the more rounded, sinusoidal structures. The second template is a typical small-scale structure taken from actual NLC data and the base for the remaining artificial templates. As the second template is not symmetric in time, it is also mirrored horizontally. The remaining templates consist of two straight lines at a $90^{\circ}$ angle, forming a wave structure with a sharp angle. The vertical brightness profile is given by a Gaussian for all templates except the second template. For the first templates, this Gaussian decreases to a background of brightness 0 . For the templates 4 and 5 the background either above or below the structure is set to $\frac{1}{3}$. These two templates are well suited for thicker layers or structures at the lower or upper edges of the NLC where the brightness decreases sharply to one side and only slightly to the other. They are also needed to detect cases where multiple layers are close to each other, such that the brightness close to the main layer is enhanced. The set of templates was chosen based on the specific wave structures that should be detected.

Slight variations of the templates, notably the layer width and the background brightness, change the amount of structures that will be found. The set presented here is chosen to minimize the number of false detections, at the cost of not identifying all structures identified visually.

\section{Template sizes}

We have investigated the effect of the template size by starting with template sizes varying in width between 2 and 30 minutes and in height between 160 and $2400 \mathrm{~m}$. The lower boundary is given by the resolution of the data, such that the template consists of at least $4 \times 4$ pixels. By visual inspection of 
the results for the subset of the data we concluded that at sizes smaller than $10 \times 10$ pixels the manual verification of the detected wave structures is no longer obvious. Here we therefore use only results of at least $10 \times 10$ pixels corresponding to 5 minutes and $400 \mathrm{~m}$. Wave structures longer than 30 minutes are often superposed by smaller scale structures, such that the correlation with the templates is considerably reduced and no matches are found.

\section{Cross-correlation threshold}

The threshold that determines a successful match is critical for the final results. The actual value is a compromise between the number of detections that do not resemble a wave structure and obvious wave structures that are missed. For example strongly asymmetric structures are not captured by the horizontally symmetric templates if the threshold value is to high. It should be kept in mind that the actual value depends on the templates and the preprocessing of the data. For the results presented here, the threshold was set to 0.8 , which restricts the results to the more evident wave structures.

\section{SNR threshold}

The results are further filtered depending on the signal-to-noise ratio to eliminate apparent structures caused by random noise. To eliminate false detections due to low data quality, the signal-to-noise ratio of the mean brightness $\beta_{\mathrm{P}}=\langle\beta(t, z)\rangle_{\text {pattern }}$ and the mean error $\Delta \beta_{\mathrm{P}}=\langle\Delta \beta(t, z)\rangle_{\text {pattern }}$ of each match must be above a threshold $\left(\beta_{\mathrm{p}} / \Delta \beta_{\mathrm{p}}>10 / 3\right)$. The brightness error is calculated based on the uncertainty of the signal and the background assuming a Poisson distribution and using Gaussian error propagation (e.g. Baumgarten et al., 2008).

\section{Multiple matches}

Overlapping matches of slightly different sizes or different templates are reduced to a single match of the template with the highest correlation coefficient. In a first step the matches where the distance between all their borders is smaller than a certain threshold (fixed at 2 pixels, i.e. $1 \mathrm{~min}$ or $80 \mathrm{~m}$ ) are reduced to the match with the highest correlation. In a second step the remaining matches are further reduced by analyzing the difference of their locations with a threshold dependent on the size of the match. Again the match with the highest correlation coefficient is selected. Since the analysis runs through the different orientations separately, this applies only to matches of the same orientation.

Fig. 4 shows the results of this analysis for an NLC measurement of $3 \mathrm{~h}$ with several significant structures. The thin black boxes represent the outer limits of the templates that were used to identify the wave structures. The length of each box gives an approximation of the scale of the observed structure. For the data inside each box, the centroid altitude is calculated, shown by the solid black line. For overlapping matches the centroid altitude (black line) might be slightly different due to the different vertical extents of the boxes. For comparison, the centroid altitude of the entire NLC data is indicated by the thin red line (interpolated 


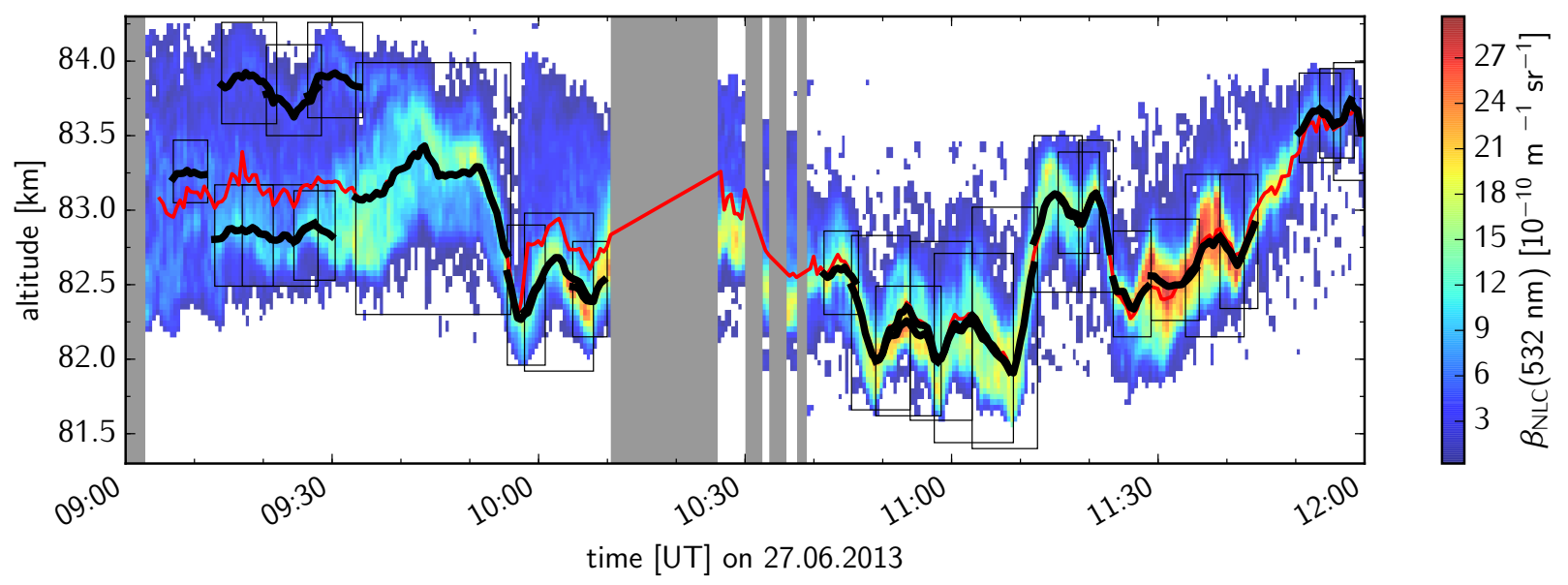

Figure 4: NLC observation on 27 June, 2013 with the results of the single pattern matching analysis: the thin black boxes indicate the outer limit of the templates that have been used to identify the structures. The thick black lines show the centroid altitude limited to the regions where patterns were identified. The global centroid altitude for the entire NLC is shown by the thin red line (interpolated over the data gaps shown by the gray bars). In total 27 structures have been found.

over the data gaps). In the second part of the NLC, the centroid altitude of the NLC closely matches the centroid altitude given by each identified pattern. Before 09:30 UT, a second structured layer at the upper edge of the NLC is detected, while the centroid altitude is between both layers.

\subsection{Method evaluation}

Previously the NLC data have been analyzed using the well known wavelet analysis (e.g. Kaifler et al. 2013). Implementations of the wavelet analysis are typically based on a Fourier transform and thus require a continuous time series (Torrence and Compo, 1998). The centroid altitude represents best the vertical oscillations in the NLC, though it may be a misleading representation if the vertical structure of the NLC is more complex. Kaifler et al. (2013) used a sophisticated scheme to separate the different layers. For the following discussion we will instead use examples of NLC where only a single layer is present. The results from the wavelet method are used as a benchmark for our new method of identifying (quantifying and locating) wavelike structures in NLC by looking at two different cases. We used a Morlet wavelet of order 5 and have applied a correction of the power spectra as suggested by Liu et al. (2007) and Chen et al. (2016) to account for a bias in the power spectra towards larger scales. It is worth noting that this does not affect the confidence limit as pointed out by Liu et al. (2007).

Two examples of NLC observations with their wavelet spectrum and the results from the detection algorithm are shown in Fig. 5. For the NLC on June 5, 2013 (Fig. 5a) the strong oscillations with periods of 2-20 min around midnight and around 2:20 UT are locally significant in the wavelet spectrum. The wavelet 

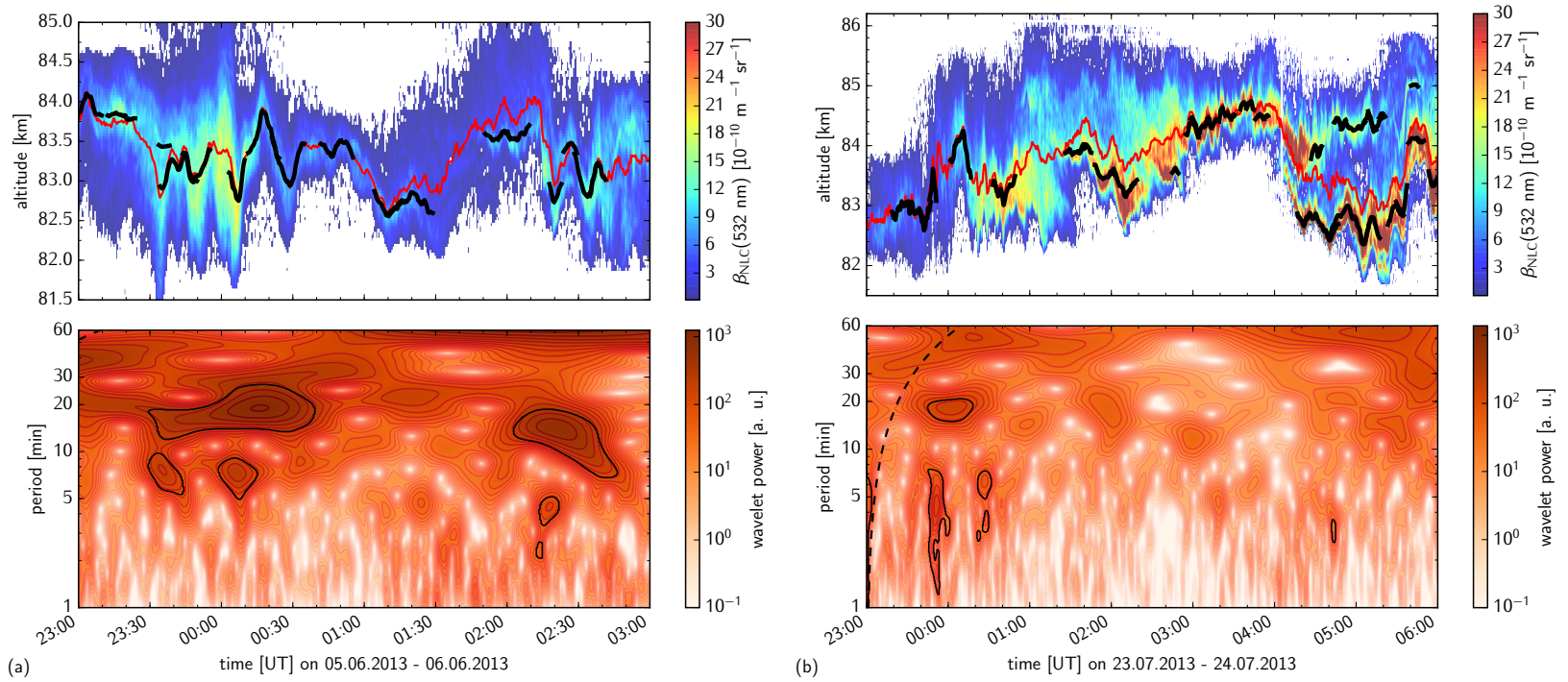

Figure 5: Upper panels: NLC observation on June 5-6 2013 (a) and on July 23-24, 2013 (b, see also Fig. 1). The red line indicates the centroid altitude, the black line indicates the centroid altitude at times where wave structures were identified by pattern recognition. Lower panels: Wavelet power spectrum of the centroid altitude (upper panels, red line) for identified continuous NLC observations. Dashed black lines indicate the cone of influence of each block of continuous NLC observation and solid black lines the 95\% significance regions after Torrence and Compo (1998).

power spectrum provides in this case information about the periods of the wave pattern and the superposition of waves at different periods. The same wave structures are also detected by the pattern recognition with a structure length between 5 and 20 min.

However, in the NLC on July 24, 2013 (Fig. 5b), there are many small structures identified by the new algorithm that are not significant in the wavelet spectrum. The wavelet analysis is performed only to the centroid altitude, in which the structures are reduced due to the large vertical extent and the multiple layers of the NLC. But also at times where the centroid altitude closely follows the wave structures as between 3:00 and 4:00 the long train of many subsequent small structures of 5-10 $\mathrm{min}$ is not significant in the wavelet spectrum.

It should be noted that several changes could improve the results of the traditional wavelet analysis: For example the choice of a different wavelet, or the reduction of the wavelet order that is better adapted to short waves, however this requires a re-evaluation of the confidence interval that was given by Torrence and Compo (1998). Considering the power spectrum, the confidence interval does not seem an appropriate measure to identify regions of wave presence on a local scale (Kaifler et al., 2013). With the parameters used here, the template matching approach seems more sensitive to the small structures than the wavelet analysis.

Kaifler et al. (2013) used local and global (i.e. time average of local spectra over an NLC observation) 
wavelet spectra for NLC data of at least $2 \mathrm{~h}$ to analyze the data acquired between 2008 and 2011 with the ALOMAR RMR Lidar. To study the significance of periods found in NLC using the significance test according to Torrence and Compo (1998) they compared the spectra of NLC observations with the spectra of random time series of similar length as the observations (Fig. 8 in Kaifler et al. (2013)). They found that in the local power spectra a large number of periods below $30 \mathrm{~min}$ are classified as significant for the random time series. This false identification of significant periods was not the case for the global spectra, which they therefore used instead.

In summary, the new pattern recognition method offers a tool to identify the local small-scale wave structures in NLC. While it depends on empirically chosen parameters, a reasonable set of parameters results in a wave structure detection very sensible to waves of only a few cycles. Due to its the image based approach it handles the two-dimensional NLC data and inherently handles multiple layers directly instead of relying on a centroid altitude. An improvement of the period estimation is possible by combining multiple adjacent matches. Other characteristics of the wave structures, as the position of the wave structure in time and altitude, is determined directly from the pattern recognition results. It provides however only an estimate of their period compared to a wavelet power spectrum, since the matched templates does not match necessarily a half or full period. Although there is a large uncertainty when directly determining the period based on the pattern recognition, the new method can be used to detect locations of interesting wave features in the large data set or provide an indication when the centroid altitude does not sufficiently represent the NLC layer due to multiple layers.

\section{Results}

The analysis was applied to the data set from 2008 to 2015 with a high temporal resolution of $30 \mathrm{~s}$. 4990 wave structures with a length between 5 and 30 min were detected in the $2000 \mathrm{~h}$ of NLC measurements between 20 May and 30 August. This corresponds to a total of $738 \mathrm{~h}$ of structures or $37 \%$ of the NLC measurements, with some of them however occurring at the same time in multiple layers. The different templates match the structures at different rates: The first, rounded template matches in $3 \%$ of all detected structures, the second template and the mirrored template both in $17 \%$, the third template in $33 \%$, the fourth in $20 \%$ and the fifth in $10 \%$. Both, the second (and its horizontally mirrored version) and the third template are the best matching templates, they are responsible for $67 \%$ of the matches. It shows that on the small scales considered here the sharp angle is characteristic, contrary to the smoother sinusoidal shape (Template 1) observed at scales of hours.

Each structure is characterized by its length corresponding approximately to the period of a wave structure. Fig. 6 shows a histogram of the occurrence and the absolute number of the length of the template. Most structures are found at a template length of $5 \mathrm{~min}$. Then the number of structures steadily decreases 


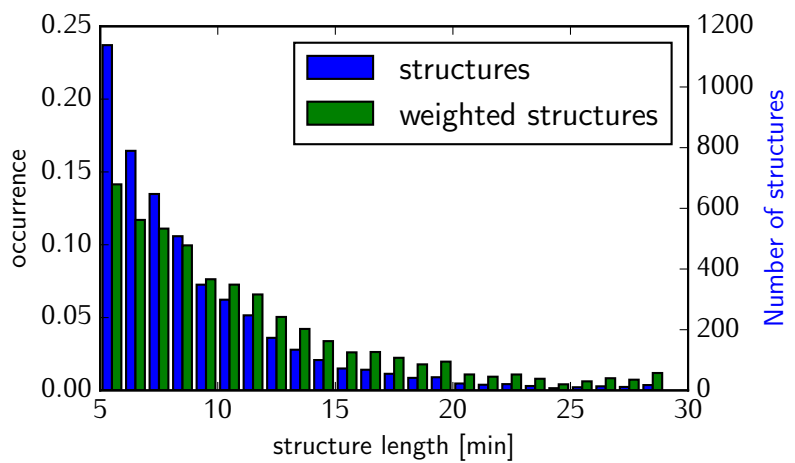

Figure 6: Number of structures by length of the template in the NLC observations from 2008 to 2015 . In blue the absolute number and the occurrence of the structures. The occurrence is defined as the fraction of the dataset found in a given structure length interval. In green the occurrence of structures weighted with their length.

towards the upper limit at $30 \mathrm{~min}$. Part of this decrease might be due to the fact that longer structures fit less often in a given time interval than shorter structures. In other words, short structures naturally may occur more often than long structures during a fixed time interval. To compare the occurrence of structures of different lengths independent of this effect, each structure is weighted with its length. This means that we look at the duration that structures of a certain length appear in total in the data set, as opposed to only the number of occurrences. After this weighting a single structure of $20 \mathrm{~min}$ has the same "duration" as 4 structures of 5 min each. This total duration compared to the total time that structures were detected $(730 \mathrm{~h}$ ) is also shown in Fig. 6 (green bars). In this representation the maximum is still located at the smallest structures, however longer structures get more important and the predominance of the shortest structures is less pronounced. It is worth noting that we do not observe a significant reduction of structures of the shortest scales of $5 \mathrm{~min}$, as we would expect for bad signal-to-noise data. Studies of even higher resolved data show that structures of $5 \mathrm{~min}$ and below exist in the data (Kaifler et al., 2013; Fritts et al., 2016).

Fig. 7f shows the distribution of NLC centroid altitude of all NLC (with and without structures) compared to the altitude of the structures detected by the pattern recognition. For the wave patterns we take the centroid altitude of wave structure only, as shown in Fig. 4. The mean centroid altitude is $83.1 \mathrm{~km}$ (standard error of the mean $\left.\sigma_{m, z_{\mathrm{c}}}=3 \mathrm{~m}\right)$ while the patterns are found on average $400 \mathrm{~m}$ lower at $82.7 \mathrm{~km}\left(\sigma_{m \text {,pattern }}=\right.$ $4 \mathrm{~m})$. Fig. $7 \mathrm{~b}$ compares the altitudes only at times when structures were found. For that case the mean NLC centroid altitude shifts to $82.8 \mathrm{~km}$, thus reducing the altitude difference to $100 \mathrm{~m}$. Finally Fig. 7f shows the distribution of the difference between the NLC centroid altitude and the structure centroid altitude for each time when a structure was detected. The distribution is slightly asymmetric and shifted towards a negative difference. 


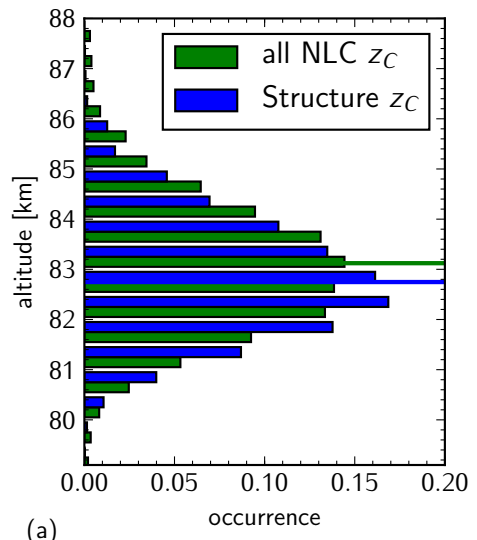

(a)

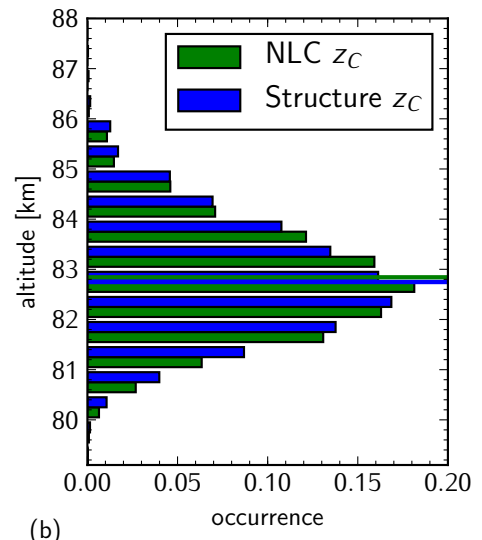

(b)

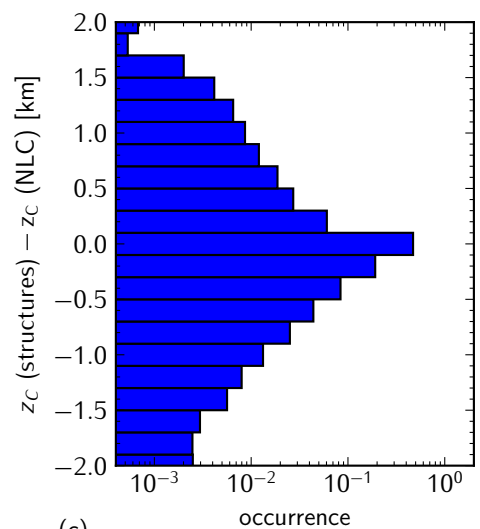

(c)

Figure 7: Histogram of the centroid altitude of the NLC (green) and of the detected wave structures (blue) for the NLC data from 2008 to 2015 using (a) all NLC data $(2000 \mathrm{~h}$ ) or (b) only the NLC data while structures were detected (738 h). The occurrence is defined as the fraction of the dataset found in a given altitude interval. The solid lines indicate the mean values. Panel (c) shows the difference between the centroid altitude of the structure to the centroid altitude of the NLC.

\section{Discussion}

\subsection{Wave structure length}

All other measurements investigating small scale structures in NLC rely on images of the clouds, provided by ground or space based camera observations and deduce horizontal wavelengths of the brightness structures. To compare to these measurements, a horizontal scale has to be derived from the length of the structures obtained by our pattern recognition method. The variations observed with the lidar are the result of local changes of the NLC as well as the advection of the NLC through the measurement volume by the background wind. It is not possible to separate these two effects using only the data from the lidar. However, Baumgarten et al. (2012) compared the lidar data with simultaneous measurement from the CIPS satellite and concluded that for structures below 30 minutes the advection is the predominant factor in the observed changes and NLC can be considered as a passive tracer on these time scales. Since our analysis is limited to periods shorter than $30 \mathrm{~min}$, we estimate a horizontal scale for the structures based on the background wind. The mean background wind (as measured by the radar) during each structure is used to calculate a corresponding horizontal scale. The resulting distribution is shown in Fig. 8. For all detected structures, the maximum lies between 10 and $20 \mathrm{~km}$. The distribution is influenced by the large number of very small structures of only 5-6 min. For these short structures the horizontal scales range most often from 5 to $15 \mathrm{~km}$. For all structures longer than $6 \mathrm{~min}$, the peak of the distribution is shifted towards $15-20 \mathrm{~km}$.

It should be noted that this horizontal scale is affected by a number of uncertainties: The scale relies on the template length which can correspond to a half up to a full period. From this we assume an uncertainty of a factor of 2 in the horizontal scales. Additionally, the wind data from the meteor radar is measured in 


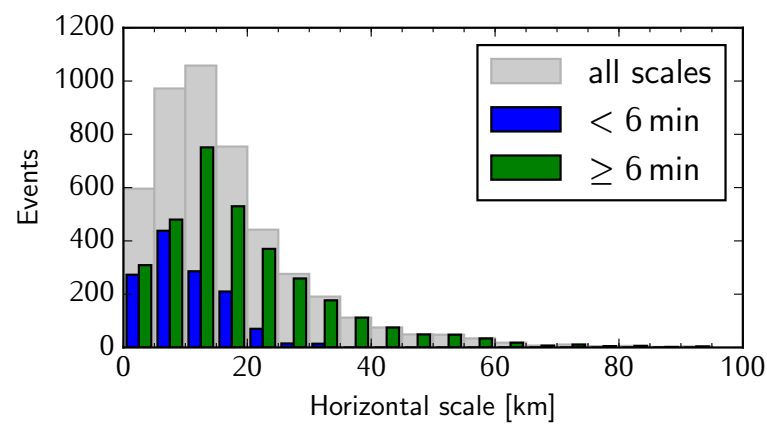

Figure 8: Number of structures of corresponding horizontal scale, based on advection by the background wind. Shown in gray is the distribution for all structures. The blue bars show the horizontal scales only for structures shorter than 6 min and the green bars for the remaining structures larger and equal to $6 \mathrm{~min}$.

a much larger volume and may not represent the actual speed of advection above the lidar, especially in context of the minute timescales that we investigate. Given these uncertainties the comparison to other data sets of horizontal scales (Fig. 9p shows a surprisingly good agreement. Pautet et al. (2011) and Demissie et al. (2014) both identified the distance between two wave crests in camera observations of NLC and found most structures at $20-30 \mathrm{~km}$ and $10-25 \mathrm{~km}$, respectively (Fig. 9). Since the camera observations are limited to the time of day where NLC are visible, the data set is much smaller. Included in Fig. $9 \mathrm{a}$ are also airglow measurements at polar latitudes which show a high occurrence of waves with horizontal wavelengths around 15-20 km (e.g. Nielsen et al., 2009 , at $76^{\circ} \mathrm{S}, 27^{\circ} \mathrm{W}$ ). These measurements are performed during the winter as the airglow can not be observed in the polar summer due to permanent daylight. Due to different wind filtering of gravity waves in the summer and winter atmosphere, wave characteristics may differ for both seasons. The analyses of observations from the CIPS instrument onboard the AIM satellite by Taylor et al. (2011) and Chandran et al. (2009) show a large number of wave structures around 20-40 km and a decrease to larger wavelengths. Later studies of the CIPS data revealed a maximum at $250 \mathrm{~km}$ (Chandran et al. 2010) or 300-800 km (Zhao et al., 2015) depending on the analysis used and the wavelengths of interest. All horizontal scales from satellite observations are far larger than the wavelengths extracted from the lidar data. The small scale structures below $40 \mathrm{~km}$ can not be clearly resolved in the satellite data due to their resolution of $5 \times 5 \mathrm{~km}$, but the large field of view of the satellite images allows for the study of much larger wavelengths. Stober et al. (2013) analyzed gravity wave events in PMSE observations with the MAARSY radar and found horizontal wavelengths around $20-50 \mathrm{~km}$.

\subsection{Wave structure altitude distribution}

Addressing the altitude distribution of the identified structures, it is remarkable that in the mean structures are observed at lower altitudes compared to the mean centroid altitude of the NLC (see Fig $7 \mathrm{a}$ ). There are a number of mechanisms that may explain this altitude difference. Near the summer mesopause in the 

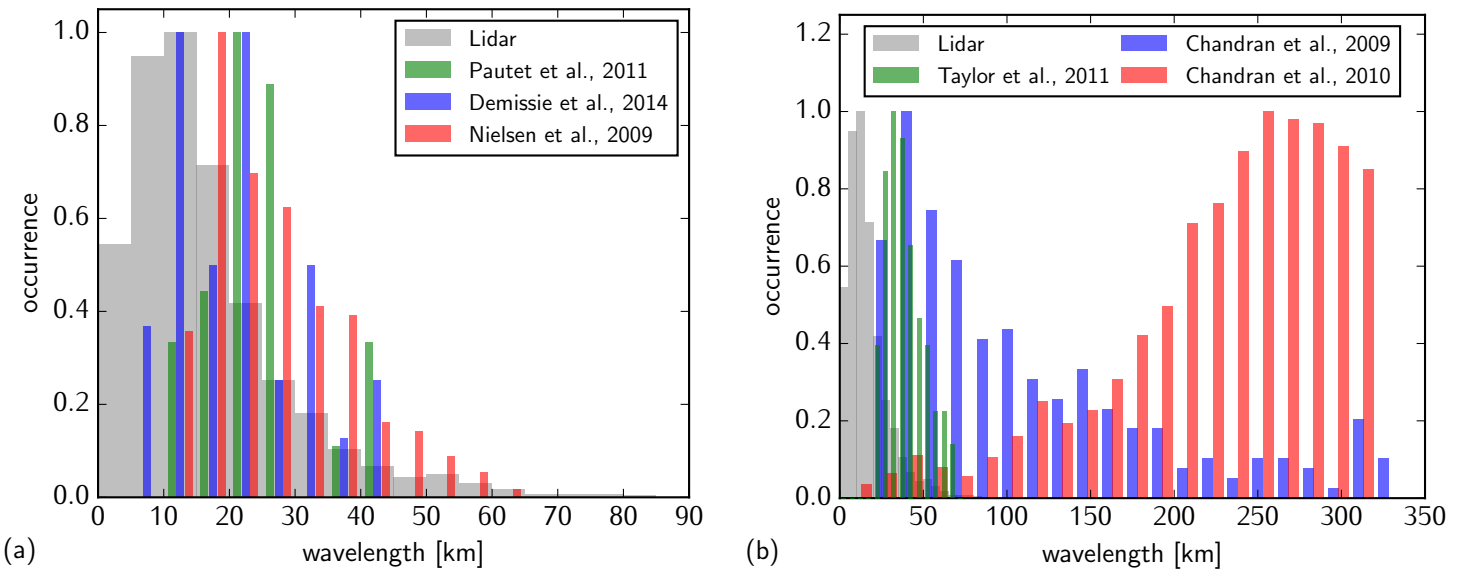

Figure 9: Horizontal scale derived from the structures in the lidar data from 2008 to 2015 (gray bars) compared to the wavelength from ground based camera and OH airglow (a) (Pautet et al. 2011 Demissie et al. 2014; Nielsen et al., 2012). Panel (b) shows the horizontal scales derived from coarser resolution satellite observations (Chandran et al., 2009, 2010 Taylor et al. 2011)

altitude region around 80-90 km atmospheric gravity waves break and produce turbulence, which maximizes at about $88 \mathrm{~km}$ and then rapidly drops towards lower altitudes (Lübken, 1997; Rapp and Thomas, 2006). This turbulence might destroy the structures in those parts of the NLC found at higher altitudes. The brightness of NLC is higher at lower altitudes (Fiedler et al., 2003; Chu et al., 2006) where the ice particles have reached their largest size (e.g. Rapp and Thomas, 2006; Baumgarten et al., 2008). With a lower brightness at higher altitudes, the lower signal-to-noise ratio reduces the chance of finding structures at these altitudes, even if they are present.

In many cases the higher brightness of the NLC at lower altitudes manifests itself in form of bright but narrow layers embedded in the "background" NLC of lower brightness (see e.g. Fig. 1 and 4). In these narrow layers the localized vertical perturbations show more clearly than in an NLC of homogeneous brightness. The difference between the altitude of the structures and the centroid altitude shown in Fig. 7 is explained as follows: The altitude of the structures indeed corresponds to the altitude of the brightest layer of the NLC. The centroid altitude is however shifted slightly upward by the small contribution of the background NLC.

The difference of $300 \mathrm{~m}$ between the altitude of structured NLC and all NLC further suggests that this difference should be taken into account when comparing the measurements with ground-based camera observations which rely on structured NLC for altitude determination. 


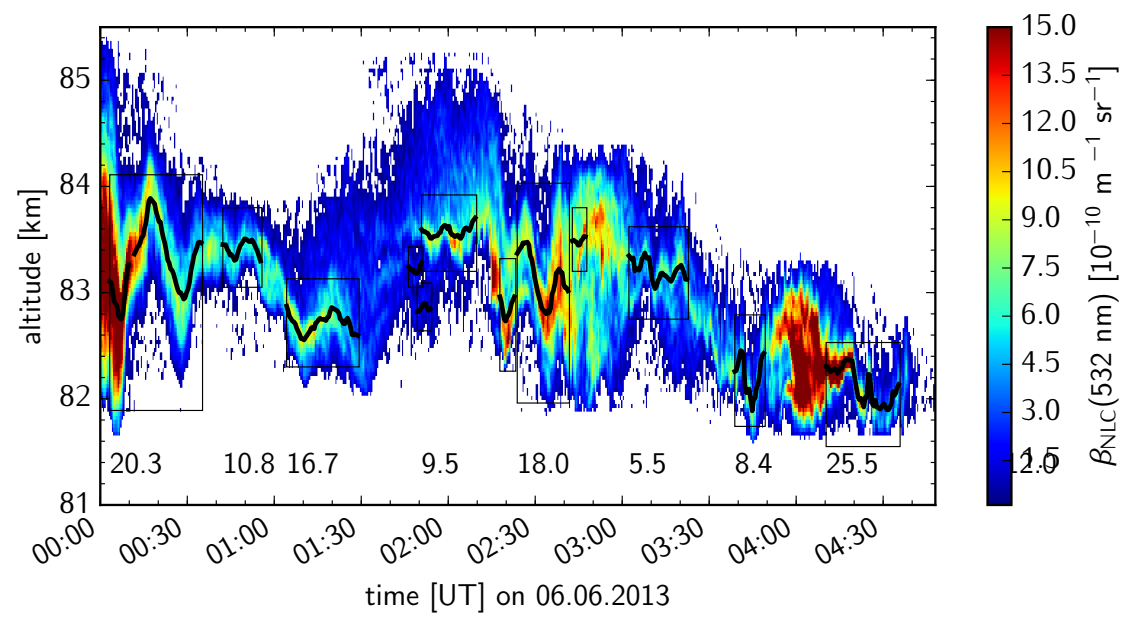

Figure 10: NLC observation on 6 June, 2013. The black lines show the centroid altitude of the identified wave patterns. Each combined wave pattern is highlighted by a frame. The period in minutes is noted below each combined pattern. Most events consist of only 2 or 3 single oscillations, before the structure disappears.

\subsection{Wave structure sequences}

Currently the pattern recognition method extracts only single oscillations from the data. Longer wave events appear as a sequence of structures, alternating between the " $V$ " and the " $\Lambda$ " orientation, sometimes overlapping. Such sequences can be merged to longer and more complex wave pattern. An example is shown in Fig. 10, where the borders of each wave pattern, consisting of combined templates, are indicated by the thin black box. From the merged wave patterns the number of oscillations and the duration of their appearance in the NLC can be determined.

The estimation of a period for the entire pattern increases the accuracy, as the period of the wave event can now be calculated using a Lomb-Scargle periodogram (Lomb, 1976; Scargle, 1982) for time series of combined centroid altitudes. That way, dominant periods of local structures embedded into a larger NLC layer can be extracted, see Fig. 10.

This analysis is based on an algorithm that identifies single subsequent wave structures which are part of the same wave event. It is crucial that the algorithm is robust, as this identification has a large impact on the derived quantities. Some of the topics to be solved prior to a statistical analysis of our large NLC data set regarding periods of merged wave events are listed in the following.

Spatial proximity as well as similarity in size of single structures are indicators for wave events. However, the complex structure of NLC layers complicates the decision if adjacent single events are part of the same larger scale period or if the observed period of the wave events varies. Difficulties also arise with the vertical distance between structures that closely follow each other. Vertical distances are often caused by vertical shifts of the entire NLC layer due to a modulation by a larger-scale wave. However, they can just as well be 
an indicator for multiple layers that feature independent wave structures. A comprehensive processing and discussion of merged wave events and their interpretation is therefore beyond the scope of this paper.

\section{Conclusion}

We have introduced an approach to analyze small-scale wave structures in lidar observations of NLC using pattern recognition. The focus lies on structures between 5 min and $30 \mathrm{~min}$, appearing only locally in the NLC. Such structures were not detectable previously by analyses based on global wavelet spectra. The measured periods of these small scale structures vary and hence do not show up significantly in the spectra. At scales longer than $30 \mathrm{~min}$, and for continuous lidar soundings without measurement gaps, a wavelet analysis is the more suited tool to identify wave events that modulate the NLC centroid altitude. However, at such longer time scales local changes of the NLC properties caused by microphysical processes become more important and the approximation of NLC particles as passive tracer is no longer valid.

The new method is sensitive to the visible small scale variations in NLC and offers a valuable tool to identify regions of strong wave activity in the large data set. The method uses directly the two-dimensional character of NLC data, allowing also for the analysis of the altitude of wave structures.

Applying the method to the ALOMAR RMR lidar NLC data set from 2008 to 2015 we calculated that wave structures with lengths between 5 and 30 min were found during $37 \%$ of the $2000 \mathrm{~h}$ of NLC data. Structures occur often within the lower part of the clouds and their mean centroid altitude is a few hundred meters below the one determined from the entire cloud altitude range. The frequency distribution shows a maximum at short structure lengths with a tail to larger values. In terms of horizontal scale, the peak of the distribution is found around $15 \mathrm{~km}$. We have demonstrated that sequences of single oscillations found by the pattern recognition method can be combined to longer wave patterns. Further investigations will be a subject of future work. Furthermore, the method itself could be used to analyze wave events in general applications having a structured tracer distinguished from a background.

\section{Acknowledgments}

We gratefully acknowledge the support of the ALOMAR staff helping to accumulate the extensive data set of NLC observations. A large number of dedicated lidar operators supported the observations during the NLC season. We are thankful to J. Hildebrand for extensive and fruitful discussions. This project has received funding from the European Union's Horizon 2020 Research and Innovation program under grant agreement No 653980 .

\section{References}

Backhouse, T. W., 1885. The luminous cirrus clouds of June and July. Meterol. Mag. 20, p. 133. 
Baumgarten, G., Chandran, A., Fiedler, J., Hoffmann, P., Kaifler, N., Lumpe, J., Merkel, A., Randall, C. E., Rusch, D., Thomas, G., 2012. On the horizontal and temporal structure of noctilucent clouds as observed by satellite and lidar at ALOMAR (69N). Geophys. Res. Lett. 39 (1).

URL http://dx.doi .org/10.1029/2011GL049935

Baumgarten, G., Fiedler, J., Lübken, F.-J., von Cossart, G., 2008. Particle properties and water content of noctilucent clouds and their interannual variation. J. Geophys. Res. 113.

Baumgarten, G., Fricke, K. H., von Cossart, G., 2002. Investigation of the shape of noctilucent cloud particles by polarization lidar technique. Geophys. Res. Lett. 29 (13), 8-1-8-4. URL http://dx.doi.org/10.1029/2001GL013877

Baumgarten, G., Fritts, D. C., 2014. Quantifying Kelvin-Helmholtz instability dynamics observed in Noctilucent Clouds: 1. methods and observations. J. Geophys. Res. 119, 9324-9337.

Bishop, C. M., 2006. Pattern Recognition and Machine Learning. Springer-Verlag New York, Inc.

Brunelli, R., Poggio, T. A., 1993. Face recognition: Features versus templates. IEEE Trans. Pattern Anal. Mach. Intell. 15, 1042-1052.

Chandran, A., Rusch, D. W., Merkel, A. W., Palo, S. E., Thomas, G. E., Taylor, M. J., Bailey, S. M., Russell III, J. M., 2010. Polar mesospheric cloud structures observed from the cloud imaging and particle size experiment on the Aeronomy of Ice in the Mesosphere spacecraft: Atmospheric gravity waves as drivers for longitudinal variability in polar mesospheric cloud occurrence. J. Geophys. Res. 115 (D13). URL http://dx.doi.org/10.1029/2009JD013185

Chandran, A., Rusch, D. W., Palo, S. E., Thomas, G. E., Taylor, M. J., 2009. Gravity wave observations in the summertime polar mesosphere from the Cloud Imaging and Particle Size (CIPS) experiment on the AIM spacecraft. J. Atmos. Solar-Terr. Phys. 71 (3-4), 392-400.

URL http://www.sciencedirect.com/science/article/pii/S1364682608002745

Chandran, A., Rusch, D. W., Thomas, G. E., Palo, S. E., Baumgarten, G., Jensen, E. J., Merkel, A. W., 2012. Atmospheric gravity wave effects on polar mesospheric clouds: A comparison of numerical simulations from CARMA 2D with AIM observations. J. Geophys. Res. 117 (D20), d20104.

URL http://dx.doi .org/10.1029/2012JD017794

Chen, C., Chu, X., Zhao, J., Roberts, B. R., Yu, Z., Fong, W., Lu, X., Smith, J. A., 2016. Lidar observations of persistent gravity waves with periods of $3-10 \mathrm{~h}$ in the Antarctic middle and upper atmosphere at McMurdo $\left(77.83^{\circ} \mathrm{S}, 166.67^{\circ} \mathrm{E}\right)$. J. Geophys. Res. 121 (2), 1483-1502.

URL http://dx.doi .org/10.1002/2015JA022127 
Chu, X., Espy, P. J., Nott, G. J., Diettrich, J. C., Gardner, C. S., Oct. 2006. Polar mesospheric clouds observed by an iron Boltzmann lidar at Rothera $\left(67.5^{\circ} \mathrm{S}, 68.0^{\circ} \mathrm{W}\right)$, Antarctica from 2002 to 2005: Properties and implications. J. Geophys. Res. 111 (D20).

Demissie, T. D., Kleinknecht, N. H., Espy, P. J., Kaifler, N., Baumgarten, G., 2014. Characteristics and sources of gravity waves observed in NLC over Norway. Atmos. Chem. Phys. 14, 12133-12142.

Fiedler, J., Baumgarten, G., Lübken, F.-J., 2009. NLC observations during one solar cycle above ALOMAR . J. Atmos. Solar-Terr. Phys. 71 (3-4), $424-433$.

URL http://wWw.sciencedirect.com/science/article/pii/S1364682608003647

Fiedler, J., Baumgarten, G., von Cossart, G., 2003. Noctilucent clouds above ALOMAR between 1997 and 2001: Occurrence and properties. J. Geophys. Res. 108 (D8).

URL http://dx.doi.org/10.1029/2002JD002419

Fritts, D. C., Isler, J. R., Thomas, G. E., Andreassen, O., 1993. Wave breaking signatures in noctilucent clouds. Geophys. Res. Lett. 20 (19), 2039-2042.

URL http://dx.doi.org/10.1029/93GL01982

Fritts, D. C., Pautet, P.-D., Bossert, K., Taylor, M. J., Williams, B. P., Iimura, H., Yuan, T., Mitchell, N. J., Stober, G., 2014. Quantifying gravity wave momentum fluxes with Mesosphere Temperature Mappers and correlative instrumentation. J. Geophys. Res. 119 (24), 13,583-13,603, 2014JD022150.

URL http://dx.doi .org/10.1002/2014JD022150

Fritts, D. C., Wang, L., Baumgarten, G., Miller, A. D., Geller, M. A., Jones, G., Limon, M., Chapman, D., Didier, J., Kjellstrand, C. B., Araujo, D., Hillbrand, S., Korotkov, A., Tucker, G., Vinokurov, J., 2016. High-Resolution Observations and Modeling of Turbulence Sources, Structures, and Intensities in the Upper Mesosphere. J. Atmos. Solar-Terr. Phys.Submitted.

Geller, M. A., Apr. 1983. Dynamics of the middle atmosphere. Space Sci. Rev. 34 (4), 359-375.

Hansen, G., Serwazi, M., von Zahn, U., 1989. First detection of a noctilucent cloud by lidar. Geophys. Res. Lett. 16 (12), 1445-1448.

URL http://dx.doi .org/10.1029/GL016i012p01445

Hervig, M., Thompson, R. E., McHugh, M., Gordley, L. L., Russell III, J. M., Summers, M. E., 2001. First confirmation that water ice is the primary component of polar mesospheric clouds. Geophys. Res. Lett. 28 (6), 971-974.

URL http://dx.doi .org/10.1029/2000GL012104 
Hines, C. O., September 1968. A possible source of waves in noctilucent clouds. J. Atmos. Sci. 25 (5), 937-942.

Hocking, W., Fuller, B., Vandepeer, B., 2001. Real-time determination of meteor-related parameters utilizing modern digital technology. J. Atmos. Solar-Terr. Phys. 63 (2), 155-169.

Hoffmann, P., Rapp, M., Fiedler, J., Latteck, R., 2008. Influence of tides and gravity waves on layering processes in the polar summer mesopause region. Ann. Geophys. 26 (12), 4013-4022.

URL http://www . ann-geophys .net/26/4013/2008/

Holton, J. R., 1983. The Influence of Gravity Wave Breaking on the General Circulation of the Middle Atmosphere. J. Atmos. Sci. 40, 2497-2507.

Itseez, 2015. Open source computer vision library. http://opencv.org.

Jesse, O., 1885. Auffallende Abenderscheinungen am Himmel. Meteorol. Z. 2, 311-312.

Jesse, O., 1896. Die Höhe der leuchtenden Nachtwolken. Astr. Nach. 140 (11), 161-168.

URL http://dx.doi.org/10.1002/asna.18961401102

Kaifler, N., Baumgarten, G., Fiedler, J., Lübken, F.-J., 2013. Quantification of waves in lidar observations of noctilucent clouds at scales from seconds to minutes. Atmos. Chem. Phys. 13 (23), 11757-11768.

URL http://www . atmos-chem-phys .net/13/11757/2013/

Kiliani, J., Baumgarten, G., Lübken, F.-J., Berger, U., Hoffmann, P., 2013. Temporal and spatial characteristics of the formation of strong noctilucent clouds. J. Atmos. Solar-Terr. Phys. 104, 151-166.

Leslie, R. C., Jul. 1885. Sky Glows. Nature 32, 245.

Liu, Y., Liang, X. S., Weisberg, R. H., 2007. Rectification of the Bias in the Wavelet Power Spectrum. J. Atmos. Ocean. Techn. 24 (12), 2093-2102.

Lomb, N. R., Feb. 1976. Least-squares frequency analysis of unequally spaced data. Astrophys. Space Sci. $39,447-462$.

Lübken, F.-J., 1997. Seasonal variation of turbulent energy dissipation rates at high latitudes as determined by insitu measurements of neutral density fluctuations. J. Geophys. Res. 102, 13, 441-13, 456.

Lübken, F.-J., Baumgarten, G., Fiedler, J., Gerding, M., Höffner, J., Berger, U., 2008. Seasonal and latitudinal variation of noctilucent cloud altitudes. Geophys. Res. Lett. 35 (6), 106801.

URL http://dx.doi .org/10.1029/2007GL032281 
Miller, A. D., Fritts, D. C., Chapman, D., Jones, G., Limon, M., Araujo, D., Didier, J., Hillbrand, S., Kjellstrand, C. B., Korotkov, A., Tucker, G., Vinokurov, Y., Wan, K., Wang, L., Jul. 2015. Stratospheric imaging of polar mesospheric clouds: A new window on small-scale atmospheric dynamics. Geophys. Res. Lett. 42, 6058-6065.

Nielsen, K., Taylor, M. J., Hibbins, R. E., Jarvis, M. J., 2009. Climatology of short-period mesospheric gravity waves over Halley, Antarctica $\left(76^{\circ} \mathrm{S}, 2^{\circ} \mathrm{W}\right)$. J. Atmos. Solar-Terr. Phys. 71 (8-9), $991-1000$. URL http://www.sciencedirect.com/science/article/pii/S1364682609000960

Nielsen, K., Taylor, M. J., Hibbins, R. E., Jarvis, M. J., Russell, J. M., 2012. On the nature of short-period mesospheric gravity wave propagation over Halley, Antarctica. J. Geophys. Res. 117 (D5).

URL http://dx.doi .org/10.1029/2011JD016261

Pautet, P.-D., Stegman, J., Wrasse, C. M., Nielsen, K., Takahashi, H., Taylor, M. J., Hoppel, K. W., Eckermann, S. D., 2011. Analysis of gravity waves structures visible in noctilucent cloud images. J. Atmos. Solar-Terr. Phys. 73 (14-15), 2082-2090.

URL http://www.sciencedirect.com/science/article/pii/S136468261000177X

Rapp, M., Lübken, F.-J., Müllemann, A., Thomas, G. E., Jensen, E. J., 2002. Small-scale temperature variations in the vicinity of NLC: Experimental and model results. J. Geophys. Res. 107 (D19), AAC 11-1-AAC 11-20.

URL http://dx.doi.org/10.1029/2001JD001241

Rapp, M., Thomas, G. E., 2006. Modeling the microphysics of mesospheric ice particles: Assessment of current capabilities and basic sensitivities. J. Atmos. Solar-Terr. Phys. 68 (7), 715-744.

URL http://www.sciencedirect.com/science/article/pii/S1364682605003007

Scargle, J. D., Dec. 1982. Studies in astronomical time series analysis. II - Statistical aspects of spectral analysis of unevenly spaced data. Astrophys. J. 263, 835-853.

Stober, G., Sommer, S., Rapp, M., Latteck, R., 2013. Investigation of gravity waves using horizontally resolved radial velocity measurements. Atmos. Meas. Tech. 6 (10), 2893-2905.

URL http://www . atmos-meas-tech.net/6/2893/2013/

Taylor, M., Pautet, P.-D., Zhao, Y., Randall, C., Lumpe, J., Bailey, S., Carstens, J., Nielsen, K., Russell III, J. M., Stegman, J., 2011. High-Latitude Gravity Wave Measurements in Noctilucent Clouds and Polar Mesospheric Clouds. In: Abdu, M. A., Pancheva, D. (Eds.), Aeronomy of the Earth's Atmosphere and Ionosphere. Vol. 2 of IAGA Special Sopron Book Series. Springer Netherlands, pp. 93-105.

URL http://dx.doi.org/10.1007/978-94-007-0326-1_7 
Torrence, C., Compo, G. P., Jan. 1998. A practical guide to wavelet analysis. Bull. Amer. Meteorol. Soc. 79 (1), 61-78.

Viola, P., Jones, M. J., 2004. Robust Real-Time Face Detection. Int. J. Comput. Vis. 57 (2), 137-154.

URL http://dx.doi.org/10.1023/B:VISI.0000013087.49260.fb

von Cossart, G., Fiedler, J., von Zahn, U., 1999. Size distributions of NLC particles as determined from 3-color observations of NLC by ground-based lidar. Geophys. Res. Lett. 26 (11), 1513-1516.

URL http://dx.doi.org/10.1029/1999GL900226

von Zahn, U., von Cossart, G., Fiedler, J., Fricke, K. H., Nelke, G., Baumgarten, G., Rees, D., Hauchecorne, A., Adolfsen, K., 2000. The ALOMAR Rayleigh/Mie/Raman lidar: objectives, configuration, and performance. Ann. Geophys. 18 (7), 815-833.

URL http://wWW . ann-geophys.net/18/815/2000/

von Zahn, U., von Cossart, G., Fiedler, J., Rees, D., 1998. Tidal variations of noctilucent clouds measured at $69^{\circ} \mathrm{N}$ latitude by groundbased lidar. Geophys. Res. Lett. 25 (9), 1289-1292.

URL http://dx.doi.org/10.1029/98GL00546

Witt, G., 1962. Height, structure and displacements of noctilucent clouds. Tellus 14 (1), 1-18.

URL http://dx.doi.org/10.1111/j.2153-3490.1962.tb00115.x

Zhao, Y., Taylor, M., Randall, C., Lumpe, J., Siskind, D., Bailey, S., III, J. R., 2015. Investigating seasonal gravity wave activity in the summer polar mesosphere. J. Atmos. Solar-Terr. Phys. 127, 8-20.

URL http://www.sciencedirect.com/science/article/pii/S1364682615000565 